\title{
Mask-less fabrication of nanowells using chemically reactive colloids
}

\author{
Neetu Chaturvedi ${ }^{+}$, Erik Hsiao ${ }^{+}$, Darrell Velegol*, and Seong H. Kim*
}

\section{Supporting Information (nl1037984)}

In order to confirm the proposed mechanism, control experiments were performed. A layer of $520 \mathrm{~nm}$ APSL particles was adhered to a glass coverslip. The glass coverslip with the adhered particles in DI water was heated in an autoclave oven at $120{ }^{\circ} \mathrm{C}$ for 90 minutes and sonicated in DI water for 60 minutes. In another experiment, a layer of $520 \mathrm{~nm}$ APSL particles was formed on silicon (100) wafer. The wafer with the adhered particles was heated in an autoclave oven at $120{ }^{\circ} \mathrm{C}$ for 90 minutes in $d r y$ condition. Another control experiment was performed by using amine-functionalized PSL particles instead of amidine-functionalized PSL particles. The FE-SEM images in Figure S-1 show that no etching occurred for any of the control experiments.
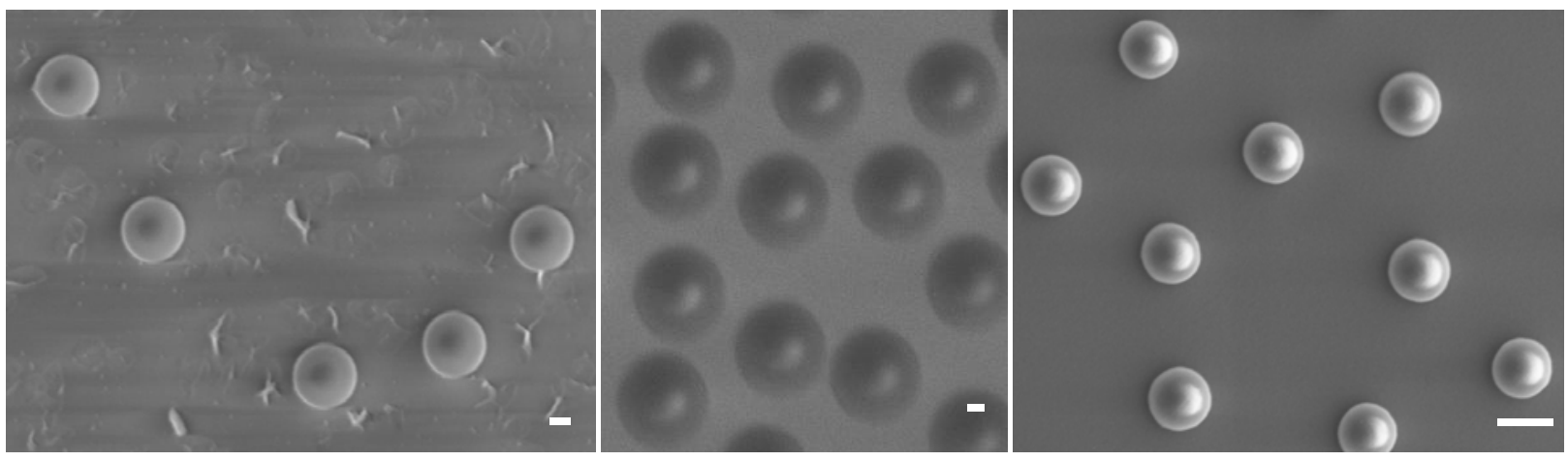

Figure S-1: Control experiments. (a) $520 \mathrm{~nm}$ APSL colloids on glass slide heated in an autoclave for 90 minutes in DI water, (b) $520 \mathrm{~nm}$ APSL colloids on silicon (100) substrate and heated in an autoclave for 90 minutes in dry conditions, (c) $200 \mathrm{~nm}$ amine-functionalized polystyrene latex colloids on silicon (100) substrate and heated in an autoclave for 90 minutes in DI water. Scale Bar $=200 \mathrm{~nm}$ 
A Kratos Analytical Ultra X-ray XPS with a $1486.7 \mathrm{eV}$ monochromatic Al Ka source was used to collect high resolution $\mathrm{N}$ 1s spectra (Figure S-2). The pass energy used was $20 \mathrm{eV}$ with a scan step size of $0.3 \mathrm{eV}$. The $\mathrm{N} 1 \mathrm{~s}$ spectra from XPS show that the amidine $\mathrm{N}^{+}$at $401 \mathrm{eV}$ is consumed by the hydrolysis from the autoclave heating at $120{ }^{\circ} \mathrm{C}$. Initially when the particles are settled onto silicon wafers, the $\mathrm{N} 1 \mathrm{~s}$ peak from the amidine group is present. This $\mathrm{N} 1 \mathrm{~s}$ peak remains up to 30 minutes of autoclave heating. After 90 minutes, the $\mathrm{N} 1 \mathrm{~s}$ peak decreases suggesting the consumption of the amidine functional group by hydrolysis. Finally after 180 minutes, all the amidine functional groups fully hydrolyze and the $\mathrm{N}$ 1s peak disappears.

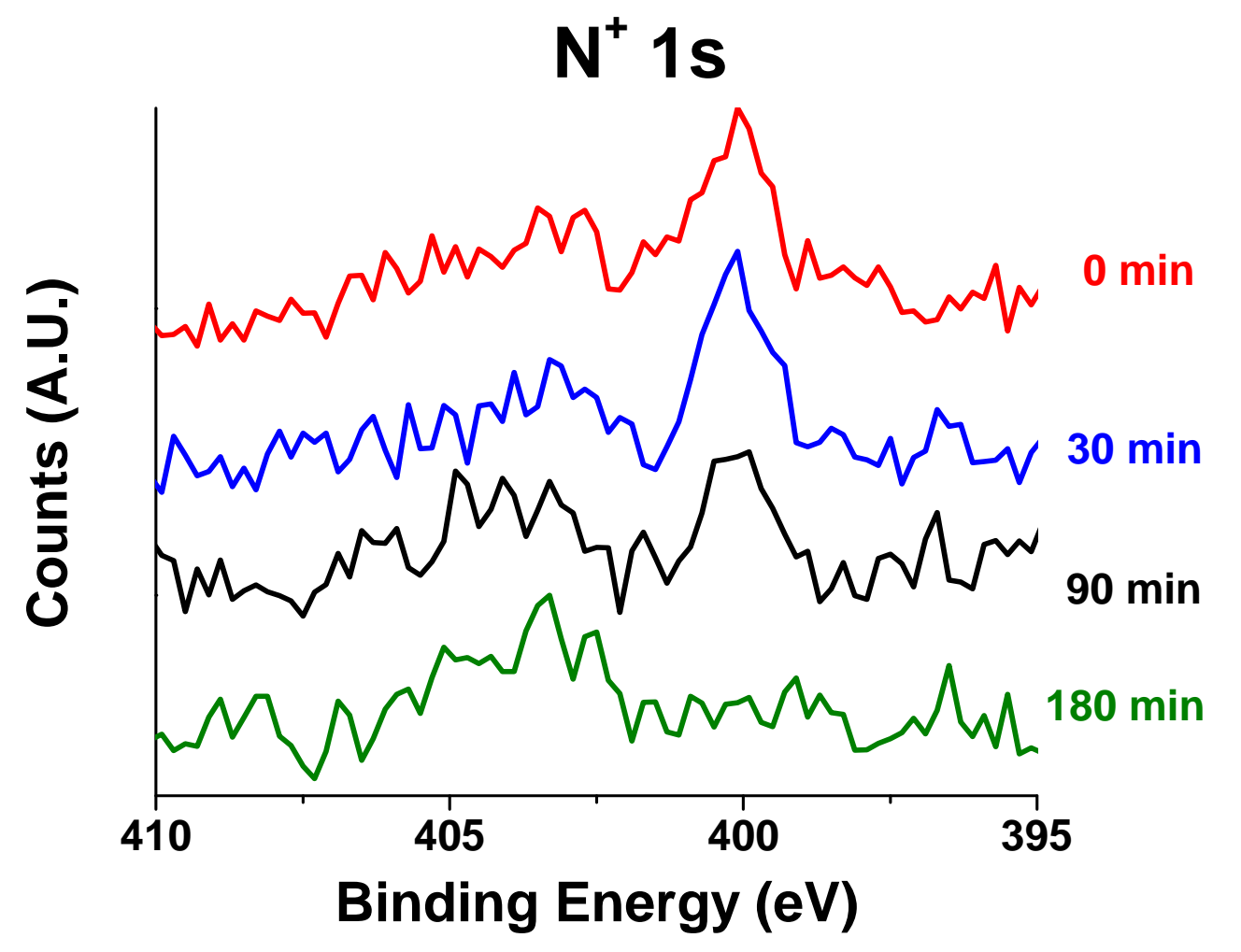

Figure S-2: N 1s XPS spectra of $520 \mathrm{~nm}$ APSL colloids on silicon (100) after 0 min, 30 min, 90 $\min$, and $180 \mathrm{~min}$ of autoclave heating. 
The Energy Dispersive X-Ray Spectroscopy (EDS) was performed on $770 \mathrm{~nm}$ APSL particles adhered onto a silicon (100) wafer. The wafer with the adhered particles in DI water were hydrolyzed at $120{ }^{\circ} \mathrm{C}$ for 120 minutes and sonicated in DI water for 60 minutes. The solution, after sonication, containing the APSL particles (as shown in Figure S-3), was collected and deposited on an aluminum stud for EDS analysis. Some aggregates were observed at the bottom of the APSL particles. The EDS result implies that these aggregates contain silicon. The aluminum shown in the EDS is due to the aluminum stud used for EDS.
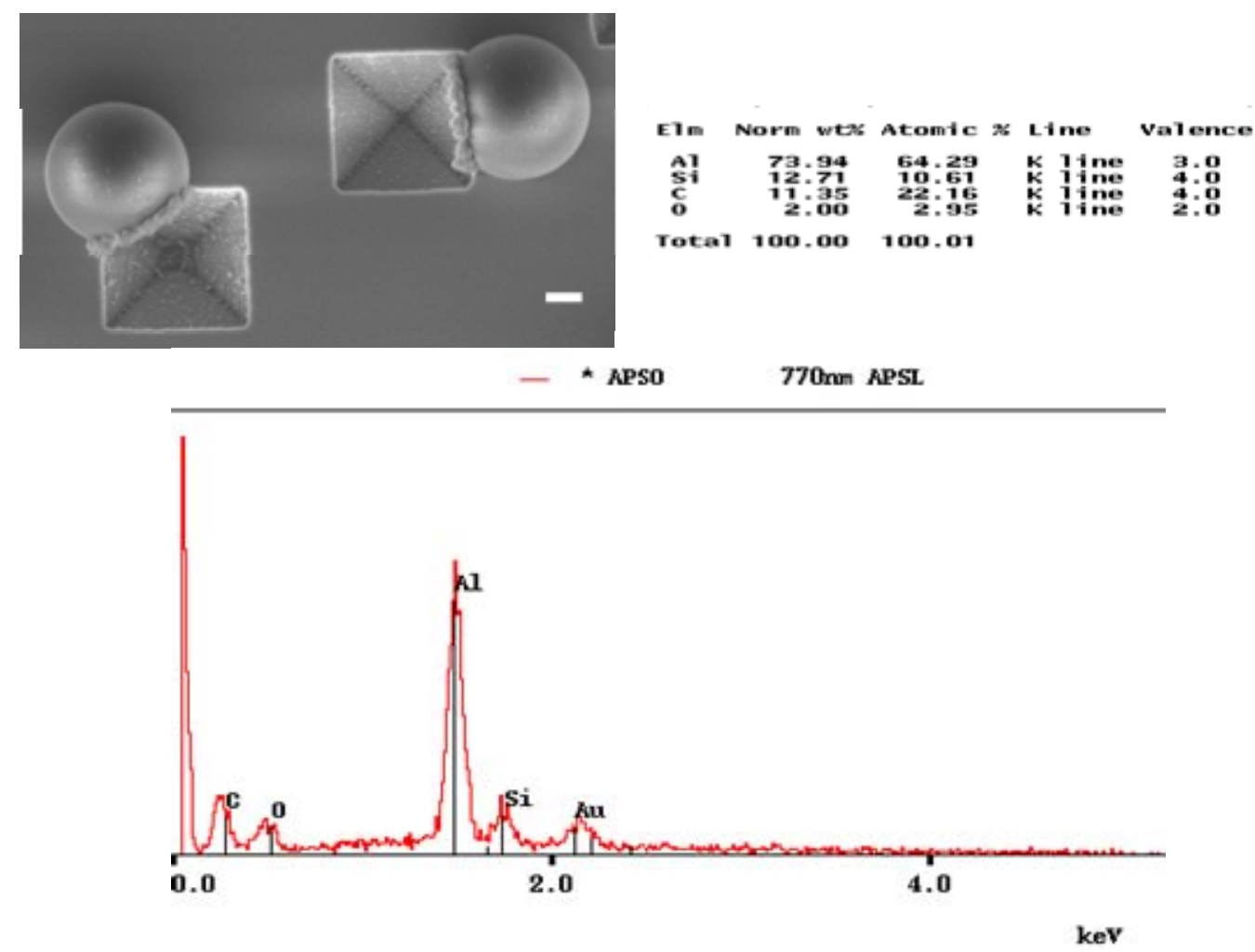

Figure S-3: SEM and EDS of $770 \mathrm{~nm}$ APSL particles after $120 \mathrm{~min}$ of autoclave heating. Scale Bar $=200 \mathrm{~nm}$.

The nanowells were formed over the entire wafer surface. The FE-SEM image in Figure S-4 shows nanowells formed over a larger area. 


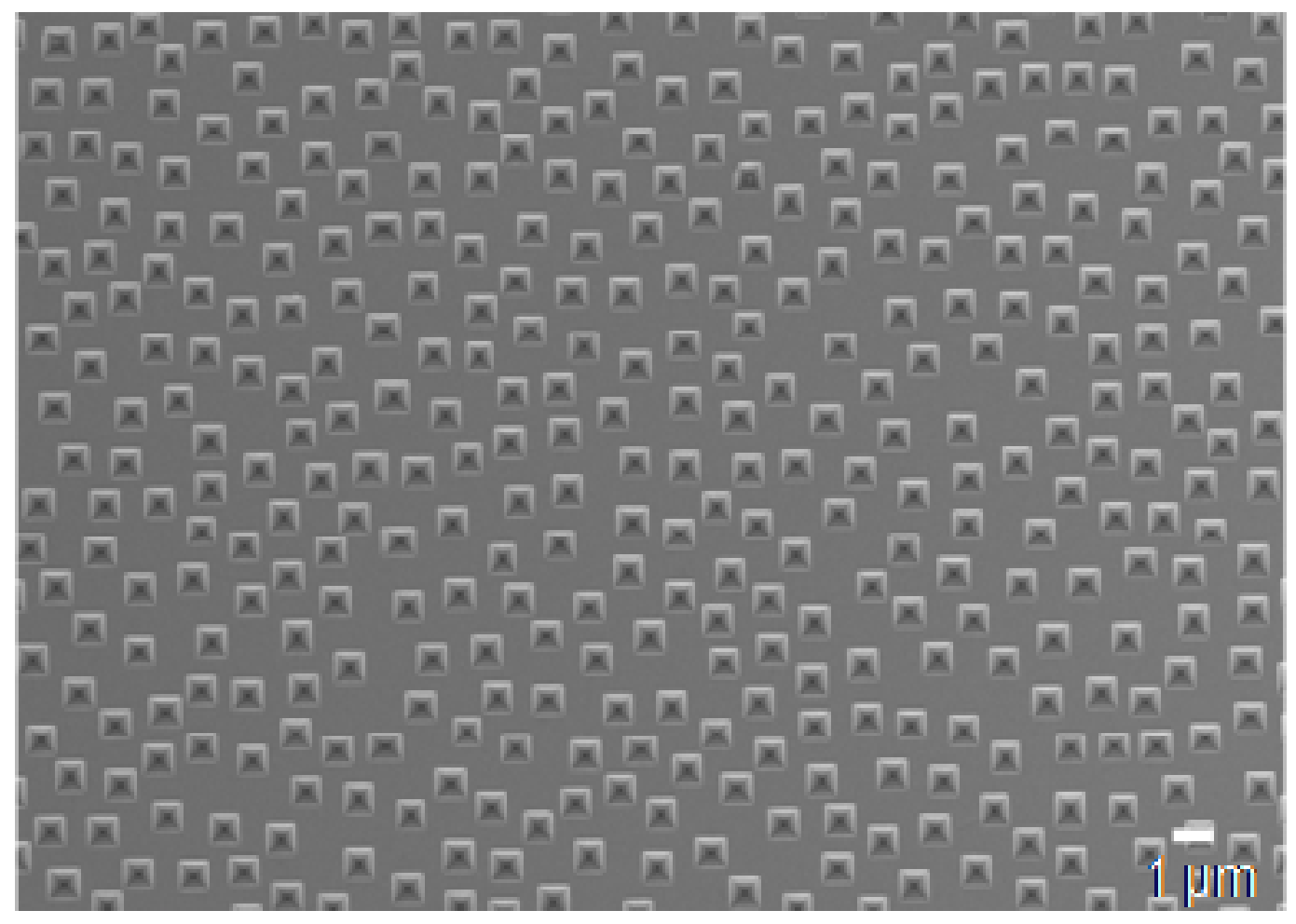

Figure S-4: Nanowells formed on a silicon (100) wafer by colloidal etching with $520 \mathrm{~nm}$ APSL particles.

$200 \mathrm{~nm}$ APSL colloids were synthesized using the protocol outlined in the literature. ${ }^{16,17}$ We used styrene as the monomer and 2, 2'-Azobis (2-methylpropionamidine) dihydrochloride as the cationic initiator. The emulsion polymerization reaction took place at $60{ }^{\circ} \mathrm{C}$ for 24 hours, forming $\sim 200 \mathrm{~nm}$ APSL particles. The cationic initiator provides the amidine functional groups on the surface of the polystyrene latex colloids. The APSL colloids were adhered to the silicon (100) wafer and heated in an autoclave for 90 minutes, which formed nanowells on the silicon wafer, as shown in Figure S-5. 

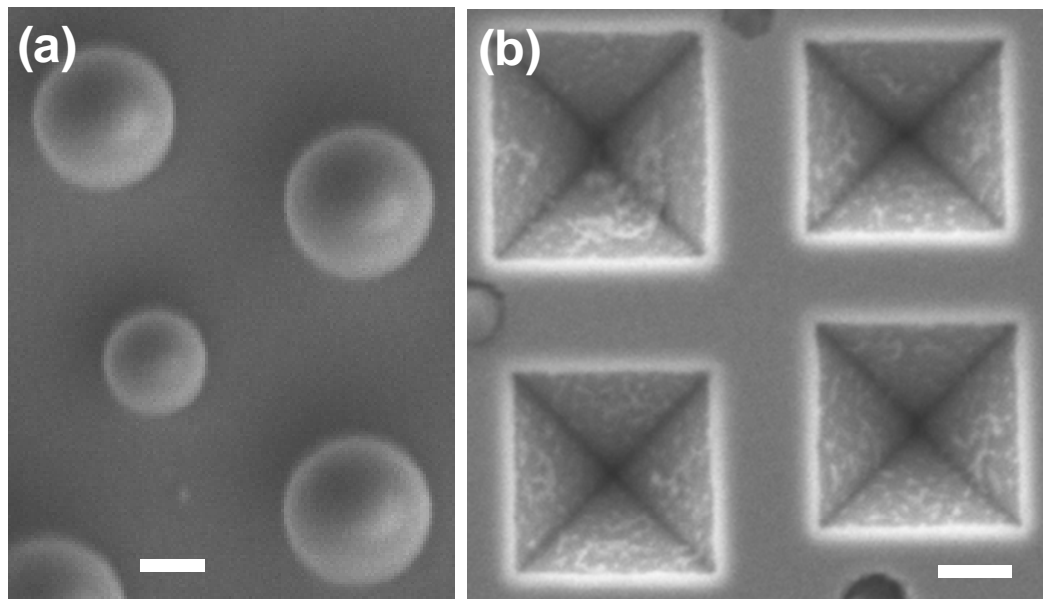

Figure S-5: (a) $200 \mathrm{~nm}$ APSL colloids, synthesized using emulsion polymerization, adhered to a silicon (100) wafer. (b) Nanowells formed by heating the synthesized $200 \mathrm{~nm}$ APSL colloids on silicon (100) wafer for 90 minutes. Scale Bar $=100 \mathrm{~nm}$. 